\title{
Estudo da influência do teor de TiC em um compósito metal duro - aço rápido para o desenvolvimento de um FGM
}

\author{
Paula, D.G.C. ${ }^{1 *}$; Alves, J.P.L.G. ${ }^{1}$; Castilho, F.G.B. ${ }^{2}$; Machado, I.F. ${ }^{2}$; Carneiro, M.B. ${ }^{1}$ \\ 1 Departamento de Engenharia Mecânica, Universidade Federal do Espírito Santo, Vitória, ES, Brasil. \\ 2 Escola Politécnica, Universidade de São Paulo, São Paulo, SP, Brasil.
}

*e-mail:daniella_gualberto@hotmail.com

\begin{abstract}
Resumo
O metal duro e o aço-rápido são dois grupos importantes de materiais para ferramenta de corte. O metal duro é capaz de compatibilizar as propriedades de dureza e tenacidade, porém este último não ocorre em nível tão elevado como no aço rápido. Material em Gradação Funcional (Functionally Graded Material FGM) é um conceito para a promoção de propriedades e/ou funções que não podem ser alcançadas por materiais homogêneos convencionais. São compósitos feitos de dois ou mais materiais com propriedades distintas na forma de um gradiente, em que as frações de volume dos constituintes variam em uma determinada direção, permitindo também a variação de suas propriedades. O objetivo do trabalho é avaliar a influência do teor de TiC sobre as propriedades mecânicas de um compósito metal duro - aço rápido, bem como analisar a microestrutura, quanto a ocorrência de segregações, a densidade relativa das amostras geradas e as características do processamento pela técnica de sinterização por corrente elétrica pulsada (PECS). Para tanto, amostras de metal duro - aço rápido com adição de TiC nas frações de volume de 5 a $35 \%$ foram sinterizadas a $1200^{\circ} \mathrm{C}$. A caracterização da microestrutura foi feita por microscopia óptica. A densidade relativa foi determinada com base no princípio de Arquimedes. A avaliação das propriedades mecânicas foi realizada por meio de ensaio de dureza Vickers e tenacidade à fratura $\left(\mathrm{K}_{\mathrm{I}}\right)$. Os resultados mostraram variação na microestrutura devido a adição de TiC, uma grande influência da pressão de sinterização na densidade relativa e nas propriedades mecânicas das amostras.
\end{abstract}

\begin{abstract}
Cemented carbide and high-speed steel are two important groups of cutting tool materials. Cemented carbide is able to match the properties of hardness and toughness; however, the latter does not occur at levels as high as in high-speed steel. Functionally Graded Material (FGM) is a concept for the promotion of properties and/or functions that cannot be achieved by conventional homogeneous materials. They are composites made of two or more materials with different properties in the form of a gradient, in which the volume fractions of the constituents vary in a certain direction, also allowing the variation of their properties. The objective of this work is to evaluate the influence of the TiC content on the mechanical properties of cemented carbide - high-speed steel composite, as well as to analyze the microstructure, regarding the occurrence of segregations, relative density of the produced samples, and the processing characteristics by pulsed electric current sintering (PECS). For this purpose, cemented carbide - high-speed steel samples with TiC addition in the volume fractions of 5 to $35 \%$ were sintered at $1200^{\circ} \mathrm{C}$. The microstructure was characterized by optical microscopy. The relative density was determined based on the Archimedes principle. The evaluation of the mechanical properties was performed by means of Vickers hardness and fracture toughness tests. The results showed microstructure changing owing to TiC addition, a great influence of the sintering pressure on the relative density and mechanical properties of the samples.
\end{abstract}

Palavras chaves: TiC, Metal duro, Aço-rápido, Dureza, Tenacidade à fratura.

\section{Introdução}

A qualidade e a consistência dos aços para ferramentas foram cada vez mais aprimoradas com o passar do tempo para o processo de fabricação de usinagem, no entanto, mesmo as melhores ferramentas em aço não foram suficientes para atender a demanda. $O$ incentivo à redução de custos, acelerando e automatizando o processo de corte, tornou-se mais intenso; e, no presente momento, atua como a principal força motriz para os desenvolvimentos tecnológicos no campo do 
corte dos metais [1]. A convergência da indústria 4.0 com as ferramentas de corte é uma tendência, impondo oportunidades de comércio, servitização, disponibilização de informação técnica via internet (IoT) e, novamente, gerando inovação ao mercado. Esse impulso ao desenvolvimento pode ser atribuído a operações industriais importantes, que envolva elevada demanda de produção em um ambiente industrial globalizado, de modo a ter-se a necessidade de produzir de maneira sustentável para se manterem competitivas no mercado diminuindo custo e aumentando a eficiência [2].

Dois grupos importantes de materiais de ferramenta de usinagem são o metal duro e o aço-rápido. O metal duro é caracterizado por partículas finas de carboneto de tungstênio (WC) que são duras, consequentemente, resistentes ao desgaste, porém frágeis e suscetíveis a trincas de origem térmica. A tenacidade à fratura é obtida através da adição de cobalto (Co), em menor quantidade, que é o ligante metálico, dúctil [3;4]. O açorápido, por sua vez, é uma liga complexa de ferrocarbono que envolve, cromo, vanádio, molibdênio, cobalto e/ou tungstênio. Caracteriza-se por elevada tenacidade, maior do que a do metal duro, o que o torna um material de ferramenta extensivamente utilizado na fabricação de brocas, fresas, brochas entre outros [5;6]. Segundo Machado et al. [7], o material de ferramenta de corte ideal deveria ter a dureza do diamante natural, a tenacidade do aço-rápido e a inércia química da alumina. Entretanto, infelizmente, ainda não foi inventado um material de ferramenta que acumulasse, simultaneamente, todas essas propriedades.

Materiais em Gradação Funcional (Functionally Graded Material - FGM) são compósitos de dois ou mais constituintes, usualmente metais e cerâmicos, formado por duas fases, no qual a fração volumétrica de ambos variam em uma determinada direção, formando um gradiente e permitindo a variação de propriedades [8;9]. Isso significa que FGMs podem ser classificados em uma categoria distinta dos compósitos homogêneos. Os perfis de transição devem ser pré-projetados e introduzidos intencionalmente para alcançar a função desejada [10].

A metalurgia do pó é um dos principais processos para a fabricação de FGMs, devido à grande disponibilidade de materiais, controle da microestrutura e da forma [10; $11 ; 12]$. Uma das técnicas de sinterização de FGM que tem recebido destaque é a sinterização por corrente pulsada (Pulsed Electric Current Sintering - PECS), também conhecida por sinterização por centelhamento (Spark Plasma Sintering - SPS). Destaca-se, principalmente, por sua capacidade de densificar materiais em alta qualidade e por curtos períodos, devido à aplicação de corrente elétrica e pressão diretamente aos pós, passando pelo molde, em geral de grafite. Promovendo, assim, o aquecimento por efeito Joule e/ou por transferência de calor durante a sinterização [12;13;14].
Segundo Braschi et al. [15], no desenvolvimento de um FGM composto por metal duro e aço-rápido foi observado a formação de fase singular de $\mathrm{Fe}$ na microestrutura, causada, provavelmente, por fenômenos de transporte de massa durante a sinterização e influenciado pelo teor de carbono no compósito. Assim, o objetivo deste trabalho é continuar o desenvolvimento prévio de um FGM através do processo de metalurgia do pó, considerando uma determinada camada com características do metal duro e do aço-rápido, agora com a adição, em diferentes concentrações, de carboneto de titânio (TiC) com elevado teor de carbono. Será avaliado o efeito dessa adição na microestrutura, a densidade relativa, a dureza e a tenacidade à fratura.

\section{Metodologia}

Braschi et al. [15] conseguiram desenvolver um FGM composto por metal duro e aço-rápido denso e isento de trincas. No entanto, observou-se algumas fases de cor escura na seção transversal da amostra, Figura 1. A análise por espectroscopia de raios $X$ por energia dispersiva (EDS) verificou que a fase escura contém, principalmente, $\mathrm{Fe}$, com presença de $\mathrm{V}$ nas bordas, sugerindo a ocorrência do fenômeno de ferritização.

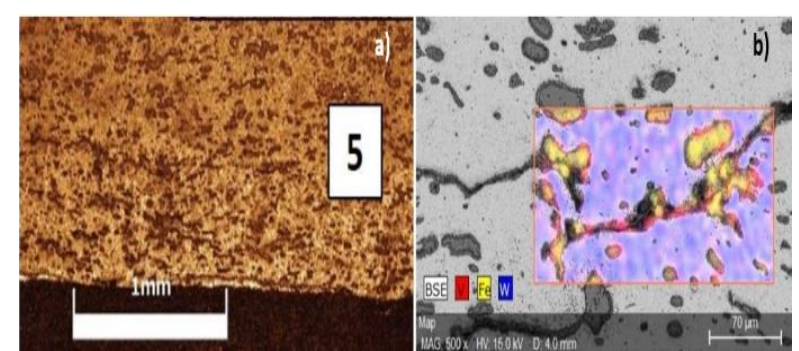

Figura 1: FGM metal duro + aço-rápido (a) Camada do FGM e (b) EDS da fase de cor escura. Fonte: [15].

Assim, pretende-se estudar o efeito da adição de frações de volume de TiC com elevado teor de carbono, em uma determinada camada do FGM (Figura 1.a), que está sendo desenvolvido pioneiramente por pesquisadores da UFES.

Os materiais utilizados são pós de metal duro (WC-Co) classe K20, fornecido pela Sandvik, carboneto de titânio (TiC), fornecido pela NanoAmor, e aço-rápido (HSS), S290, da Böhler. A Tabela 1 apresenta características dos pós.

\begin{tabular}{ccc} 
Tabela 1: Características dos materiais utilizados & \\
\hline Materiais & $\begin{array}{c}\text { Tamanho de } \\
\text { partícula }[\mathbf{\mu m}]\end{array}$ & $\begin{array}{c}\text { Densidade } \\
{\left[\mathbf{g} / \mathbf{c m}^{3}\right]}\end{array}$ \\
\hline WC-Co & 1,50 & 14,90 \\
\hline TiC & 0,08 & 4,93 \\
\hline S290 & 50,00 & 8,30 \\
\hline
\end{tabular}

De acordo com grupo Sandvik, o metal duro K20, tem a composição química (\%massa) dada por: 93,35 WC; 6,5 Co; $0,15 \quad \mathrm{Cr}_{3} \mathrm{C}_{2}$. A temperatura de sinterização convencional é $1450^{\circ} \mathrm{C}$. Da mesma forma, segundo o 
grupo Böhler, o aço-rápido S290, possui a composição química (\%massa) dada por: $2,0 \mathrm{C}$; $0,43 \mathrm{Si}$; 0,3 Mn; 0,021 P; 0,018 S; 3,77 Cr; 2,49 Mo; 4,83 V; 14,27 W; 11,0 Co; e 60,871 Fe. Temperatura de austenitização de $1210^{\circ} \mathrm{C}$.

O procedimento de mistura de pós foi desenvolvido, de modo que amostras de metal duro - aço rápido tivessem adição de TiC nas frações de volume de 5, 15, 25 e 35\%. Assim, foram definidas quatro amostras com volume de $1 \mathrm{~cm}^{3}$ cada, as frações volumétricas estão apresentadas na Tabela 2.

Tabela 2: Fração em volume de TiC e metal duro (MD) - aço-rápido (HSS) para cada amostra.

\begin{tabular}{ccc}
\hline Amostra & TiC [\%] & MD - HSS [\%] \\
\hline 1 & 5 & 95 \\
\hline 2 & 15 & 85 \\
\hline 3 & 25 & 75 \\
\hline 4 & 35 & 65
\end{tabular}

Utilizou-se uma balança de precisão, modelo AD200 (Marte), para a medição das massas e acondicionamento dos pós em diferentes frascos. Em seguida, os pós foram misturados e homogeneizados em meio líquido de álcool isopropílico por cerca de 37 horas em um agitador tipo Wagner (New Lab), fazendo uso de elementos de moagem, na proporção mássica de 2:1. Posteriormente, os pós foram secos em estufa, modelo NL80/42 (New Lab) durante 24 horas e desaglomerados mecanicamente por 6 horas.

A sinterização das amostras foi realizada em uma máquina de sinterização por corrente pulsada (PECS), modelo 1050 da SPS Syntex Inc. (Figura 2), que executa o controle do ciclo térmico e de pressão automaticamente, a partir de um controlador PID.

As condições de sinterização para cada amostra se encontram na Tabela 3. Os pós soltos foram colocados manualmente em matriz de grafite, sinterizados à vácuo, na temperatura de $1200^{\circ} \mathrm{C}$ e por um tempo de $6 \mathrm{~min}$. A taxa de aquecimento e de resfriamento foi de $100^{\circ} \mathrm{C} / \mathrm{min}$ e $80^{\circ} \mathrm{C} / \mathrm{min}$, respectivamente.
A temperatura de sinterização foi definida com base na literatura [16;17], na experiência prévia com os materiais de trabalho e além disso, a austenitização do HSS acontece por volta de $1200^{\circ} \mathrm{C}$. A Amostra 1 foi sinterizada com pressão uniaxial de $20 \mathrm{MPa}$, porém as Amostras 2, 3 e 4 devem ser consideradas, como sinterizadas sem pressão. O valor indicado na tabela de 2,2 MPa é o mínimo para o funcionamento prático da máquina. $O$ estudo variando a pressão justifica-se, pois, o HSS nas condições de teste tem formação de fase líquida intensa, e devido a isso, pode haver vazamento de material em relação ao molde.

\begin{tabular}{cccc}
\multicolumn{4}{c}{ Tabela 3: Condições de sinterização das amostras por PECS. } \\
\hline $\begin{array}{c}\text { Amostra } \\
\text { Temperatura } \\
{\left[{ }^{\circ} \mathbf{C}\right]}\end{array}$ & $\begin{array}{c}\text { Pressão } \\
{[\mathrm{MPa}]}\end{array}$ & Atmosfera \\
\hline 2 & 1200 & 20,0 & Vácuo \\
\hline 3 & 1200 & 2,2 & Vácuo \\
\hline 4 & 1200 & 2,2 & Vácuo \\
\hline
\end{tabular}

As amostras sinterizadas foram preparadas metalograficamente. Para tanto, elas foram seccionadas na transversal, utilizando uma cortadora metalográfica, IsoMet 1000 (Buehler), com disco de corte 15HC (Buehler), sendo uma parte reservada para ensaio de densidade e a outra para avaliação de propriedades mecânicas. Na sequência, embutidas com uso de resina fenólica (baquelite) em uma embutidora à quente EFD 30 (Fortel). As amostras foram lixadas com pós de SiC nas granulometrias de \#400, \#600 e \#1000. Por fim, polidas com pastas de diamantes de 15, 6 e $1 \mu \mathrm{m}$ em uma politriz PLFDV (Fortel).

Após a preparação as amostras foram levadas ao microscópio óptico Nikon Eclipse MA200 para registro e análise da microestrutura.

As densidades relativas foram determinadas com base na norma ISO 10545-3:1997 [18]. Para este procedimento, utilizou-se uma balança de precisão Marte AD200 (resolução de 0,001 g) com kit hidrostático. Primeiramente, as amostras foram aquecidas em água

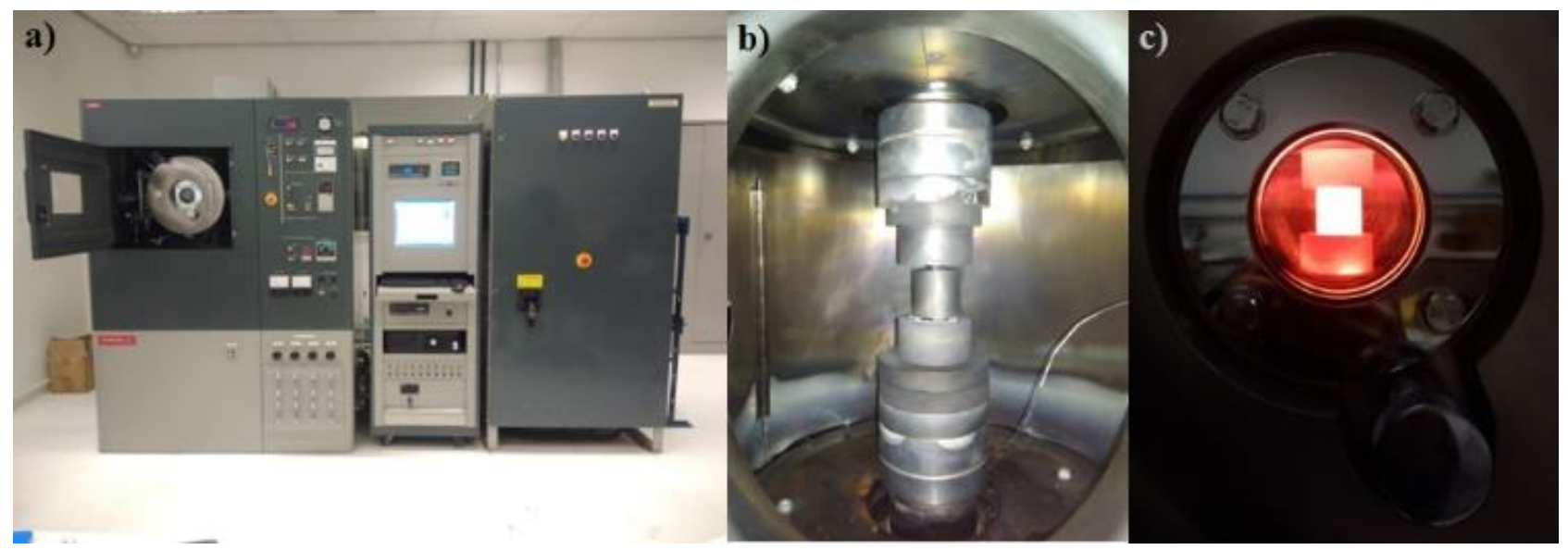

Figura 2: Processo de Sinterização. (a) Máquina de sinterização por corrente pulsada, modelo 1050 (SPS Syntex Inc.); (b) Montagem da matriz com a amostra na SPS; (c) Durante a sinterização. Fonte: Autor (2020). 
destilada até a temperatura de ebulição por duas horas (Figura 3.a) e depois resfriadas imersas em água até a temperatura ambiente por mais duas horas. Em seguida, mediu-se a massa suspensa $\left(m_{3}\right)$, utilizando-se 0 aparato de Arquimedes (kit hidrostático) da balança (Figura 3.b). As medições de massa foram realizadas com monitoramento da temperatura da água por um termômetro digital de espeto TM879 (Equitherm).

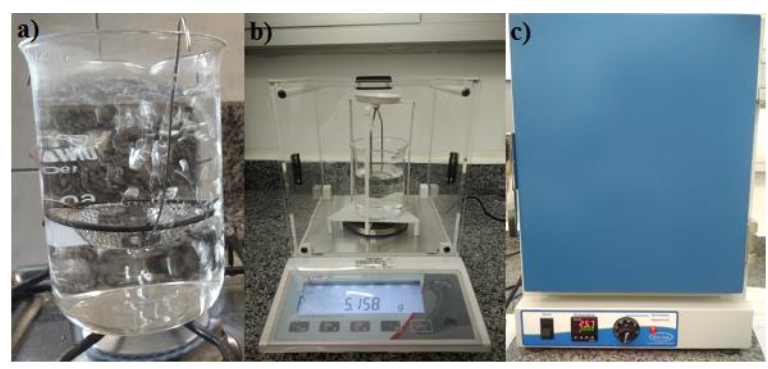

Figura 3: Aparato par medição de densidade relativa. (a) Amostra em fervura; (b) Kit hidrostático; (c) Estufa New Lab. Fonte: Autor (2020).

Na sequência mediu-se a massa úmida $\left(\mathrm{m}_{2}\right)$ retirando as amostras da água destilada e removendo o excesso de água das superfícies, antes de colocá-las na balança. Por fim, mediu-se a massa seca $\left(m_{1}\right)$ das amostras, para isso as mesmas foram mantidas em estufa a $100^{\circ} \mathrm{C}$ por 24 horas (Figura 3.c).

Para cada massa foram realizadas 15 medições para determinar a densidade experimental $\left(\rho_{\text {exp }}\right)$ mostrada na Eq. (1):

$\rho_{\text {exp }}=\frac{m_{1}}{m_{2}-m_{3}} \times \rho_{H_{2} O}$

A densidade da água destilada foi determinada pela Eq. (2):

$$
\rho_{\mathrm{H}_{2} \mathrm{O}}=1,0017-0,0002135 \times T\left[\mathrm{~g} / \mathrm{cm}^{3}\right]
$$

onde $T$ é a temperatura da água em [ $\left.{ }^{\circ} \mathrm{C}\right]$, medida durante a obtenção da massa suspensa [18]. A densidade teórica $\left(\rho_{\text {teó }}\right)$ foi obtida pela Eq. (3), pela regra de misturas [19], utilizando as densidades dos materiais na Tabela 1 e porcentagem em massa (wt\%) dos componentes.

$\frac{1}{\rho_{\text {teó }}}=\frac{w t \%_{W C-C o}}{\rho_{W C-C o}}+\frac{w t \%_{H S S}}{\rho_{H S S}}+\frac{w t \%_{T i C}}{\rho_{T i C}}$

As densidades relativas $\left(\rho_{\text {rel }}\right)$ de cada amostra resultam da relação entre as densidades teóricas e experimentais, conforme a Eq. (4).

$\rho_{\text {rel }}=\frac{\rho_{\text {exp }}}{\rho_{\text {teó }}} \times 100[\%]$

Finalmente, avaliou-se as propriedades mecânicas das amostras por meio de ensaios de dureza Vickers e tenacidade à fratura $\left(\mathrm{K}_{\mathrm{IC}}\right)$ em um durômetro Wolpert de acordo com a norma ASTM C1327 - 15:2008 [20]. Foram realizadas 12 indentações com cargas de, $31,25 \mathrm{kgf}$ para a Amostra 1 e $60 \mathrm{kgf}$ para as demais, isso foi necessário para que o comprimento das trincas fosse maior do que a diagonal da impressão. As indentações foram registradas no microscópio óptico para se avaliar a altura e a largura das diagonais, assim como a escala, a partir de ferramentas de formatação de imagem. Com tais valores calibrou-se o sistema e calculou-se as dimensões verdadeiras das diagonais, Figura 4.

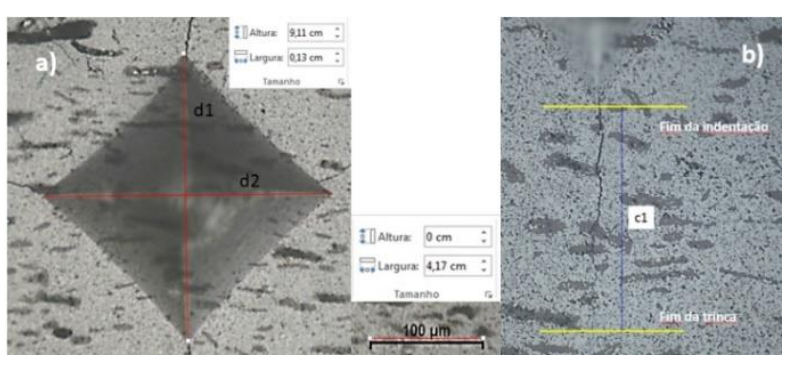

Figura 4: Método de avaliação da imagem. (a) Dureza e (b) Tenacidade à fratura. Fonte: Autor (2020).

O número de dureza Vickers foi calculado conforme mostrado na Eq. (5), usando $P$ em quilograma-força e o comprimento médio das diagonais (d) em milímetro.

$H V=1,8544\left(\frac{P}{d^{2}}\right)$.

A tenacidade à fratura por deformação plana $\left(\mathrm{K}_{\mathrm{IC}}\right)$ no modo I de carregamento, foi medida usando a Eq. (6).

$K_{I C}=0,016\left(\frac{E}{H}\right)^{1 / 2} \frac{P}{c^{3 / 2}}$

onde $E$ é o módulo de elasticidade [GPa], $H$ é o valor de dureza [GPa], $P$ é a carga aplicada [N], $c$ é a extensão da trinca [m] e $K_{I C}$ é dado em [MPa.m ${ }^{1 / 2}$ ] [21].

\section{Discussão dos resultados}

A Figura 5 apresenta as imagens obtidas pelo microscópio óptico. Pela análise das imagens, nota-se que a Amostra 1 (5\% TiC) e Amostra 2 (15\% TiC) apresentaram uma fase escura na forma de veios e esferoidizadas, respectivamente, assim como observado por Braschi et al. [15].

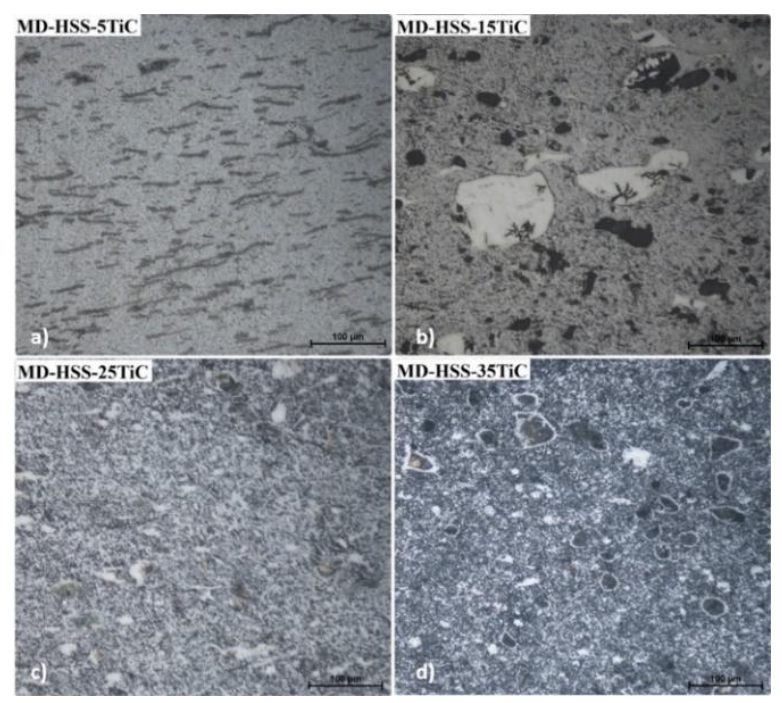

Figura 5: Micrografia das amostras aumentadas em 100 vezes. a) Amostra com 5\%TiC; b) Amostra com 15\% TiC; c) Amostra com $25 \% \mathrm{TiC}$; d) Amostra com 35\%TiC. Fonte: Autor (2020). 
A forma de veios deve-se à pressão uniaxial de sinterização, enquanto a forma esferoidizada, à ausência dela. Em ambas, a fase escura, possivelmente, seja ferro, que segregou durante os fenômenos de transporte de massa da sinterização. Na Figura 5.c, Amostra 3 (25\%TiC), tem-se uma microestrutura mais homogênea, sem presença de fase escura, o mesmo ocorre na Amostra 4 (35\%TiC), porém nesta observouse aglomerados que sugerem ser de $\mathrm{TiC}$, que não se homogeneizaram com a matriz. Nota-se, então, que o teor de $\mathrm{TiC}$ influenciou na microestrutura.

Os resultados obtidos para as densidades das amostras são apresentados na Tabela 4. Percebe-se que a Amostra 1 apresentou a maior densidade relativa, próxima a $98 \%$, e as demais apresentaram valores menores, sendo que, quanto maior o teor de $\mathrm{TiC}$, obteve-se menor densidade relativa, conforme a Figura 6. Avalia-se que a pressão exercida durante a sinterização por pulso de corrente elétrica tem grande influência na densidade relativa, visto que, para a Amostra 1, foi aplicada uma pressão de $20 \mathrm{MPa}$, cerca de 10 vezes maior do que a pressão aplicada as outras (2,2 MPa), considerado sem pressão. Ocasionando, assim, menor presença de poros na Amostra 1, o que vai influenciar nas propriedades mecânicas.

\begin{tabular}{ccccc}
\multicolumn{5}{c}{ Tabela 4: Avaliação da densidade para as amostras sinterizadas. } \\
\hline Amostra & TiC [\%] & $\begin{array}{c}\boldsymbol{\rho}_{\text {teó }} \\
{\left[\mathbf{g} / \mathbf{c m}^{3}\right]}\end{array}$ & $\begin{array}{c}\boldsymbol{\rho}_{\text {exp }} \\
{\left[\mathbf{g} / \mathbf{c m}^{3}\right]}\end{array}$ & $\begin{array}{c}\boldsymbol{\rho}_{\text {relat }} \\
{[\%]}\end{array}$ \\
\hline $\mathbf{1}$ & 5 & 11,89 & 11,624 & 97,776 \\
\hline $\mathbf{2}$ & 15 & 11,16 & 9,047 & 81,065 \\
\hline $\mathbf{3}$ & 25 & 10,43 & 8,118 & 77,831 \\
\hline $\mathbf{4}$ & 35 & 9,69 & 7,377 & 76,120
\end{tabular}

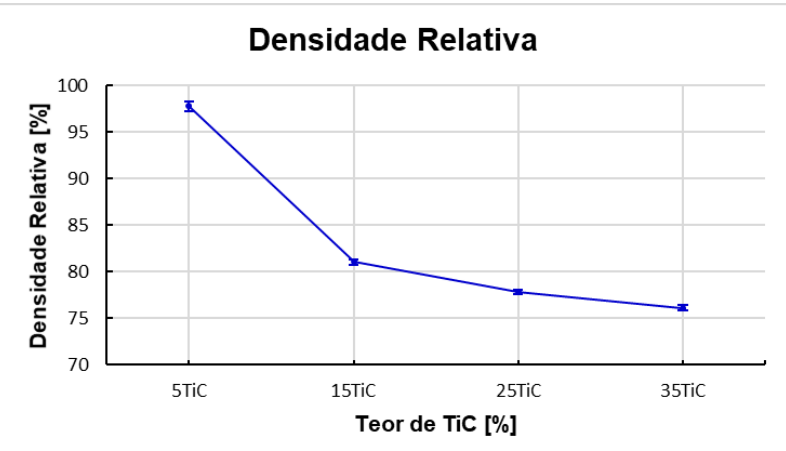

Figura 6: Avaliação da densidade relativa após sinterização por PECS. Fonte: Autor (2020).

Braschi et al. [15] conseguiram densidades relativas acima de $96 \%$, porém a sinterização ocorreu em pressão de $40 \mathrm{MPa}$ e sem a adição de TiC.

Para as amostras sinterizadas sem pressão (15\%, 25\% e $35 \% \mathrm{TiC}$ ), observou-se a diminuição de densidade relativa em relação ao aumento do teor de TiC. O resultado era esperado, pois o carboneto de titânio é um cerâmico, que necessita de maior aporte térmico para a sua sinterização, quando isso não ocorre, os efeitos de transporte de massa não são efetivos, contribuindo para a não diluição do carboneto, desprendimento do mesmo à matriz $\mathrm{e}$, consequentemente, aumento de porosidade. O estudo com e sem pressão foi importante para dar suporte a desenvolvimentos posteriores, ainda assim, permitiu evidenciar modificações microestruturais.

Segundo German [22], a densidade relativa se relaciona diretamente com as propriedades do sinterizado. Quanto mais denso o material, espera-se maior a dureza. Os resultados da dureza são apresentados na Tabela 5, ilustrado na Figura 7.

Tabela 5: Resultados do ensaio de dureza Vickers e tenacidade à fratura

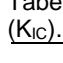

\begin{tabular}{ccccc}
\hline Amostra & $\boldsymbol{\rho}_{\text {rel }}[\%]$ & TiC [\%] & $\begin{array}{c}\text { Dureza } \\
\text { Vickers } \\
{[\mathrm{HV}]}\end{array}$ & $\begin{array}{c}\mathrm{KIC} \\
{[\mathrm{MPa}} \\
\mathbf{m}^{\mathbf{1} / 2} \text { ] }\end{array}$ \\
\hline 1 & 97,766 & 5 & 1245 & 12 \\
\hline 2 & 81,065 & 15 & 655 & 16 \\
\hline 3 & 77,831 & 25 & 454 & 7 \\
\hline 4 & 76,120 & 35 & 358 & 5 \\
\hline
\end{tabular}

\section{a)}

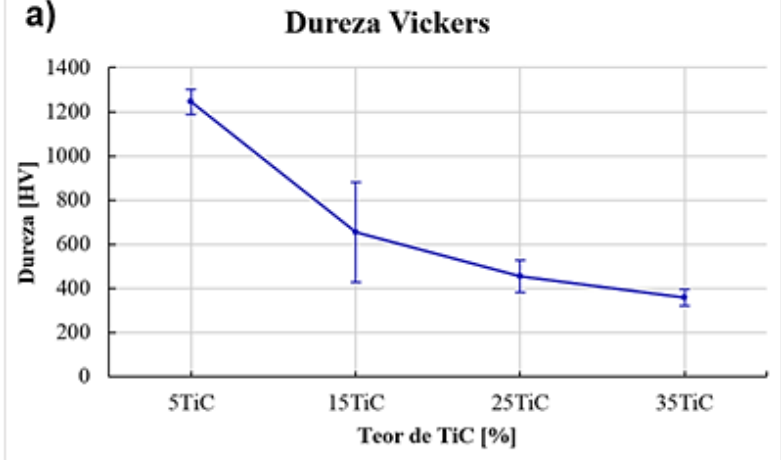

b)

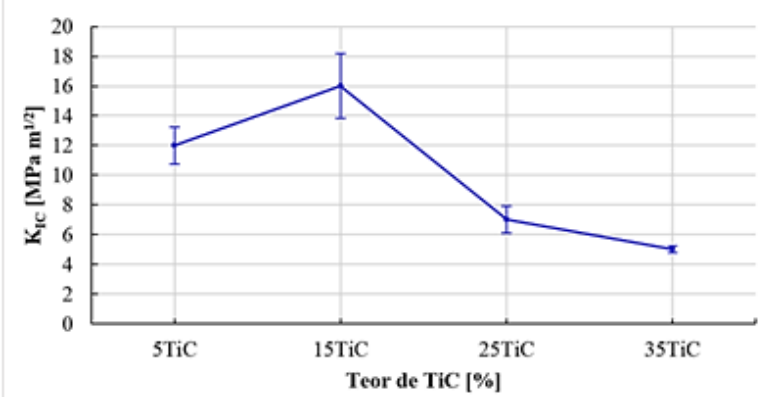

Figura 7: Gráficos de propriedades por teor de TiC. (a) Dureza e (b) Tenacidade à fratura. Fonte: Autor (2020).

A diminuição de dureza com o aumento do teor de TiC pode ser atribuída pelo aumento de porosidade nas amostras, oriunda da falta de pressão exercida durante a sinterização das Amostras 2, 3 e 4. Era esperado um aumento de dureza com aumento do teor de TiC em condições de amostras completamente densas.

A porosidade também pode ter influenciado os resultados de tenacidade à fratura. Observa-se que houve um aumento de tenacidade para a Amostra $2 \mathrm{em}$ relação à Amostra 1, isso pode ser explicado devido à 
maior presença de carbonetos na matriz. Uma maior concentração de carbonetos na matriz pode dificultar a propagação de trincas e com isso aumentar a tenacidade à fratura. Outra possibilidade é que a porosidade, geralmente, se localiza nos contornos de grão em materiais sinterizados, podendo absorver a energia de propagação da trinca ao mudar o modo de fratura, assim como influenciar no módulo de elasticidade [21;23]. O aumento de $\mathrm{K}_{\mathrm{IC}}$ associado ao aumento de TiC pode ser justificável até uma determinada composição, pois a tendência é que o carboneto duro torne o material mais frágil, propiciando uma maior propagação da trinca. O resultado das Amostras 3 e 4 sugerem que o excesso de porosidade diminui a resistência do material à propagação da trinca, como menciona German [22]. Dessa forma, o resultado não é conclusivo, para avaliar o efeito de teores mais elevados de TiC.

\section{Conclusão}

Os resultados permitiram evidenciar que os teores de TiC podem influenciar na microestrutura, de modo a deixá-las mais homogêneas e sem a presença de fases segregadas contendo ferro. Notou-se que é necessário um mínimo de pressão uniaxial para que a sinterização por PECS promova elevada densificação. Esta influencia positivamente na densidade relativa, e consequentemente nas propriedades mecânicas, sendo que dureza e tenacidade à fratura, são características importantes em uma ferramenta de usinagem. Observou-se que a grande concentração de poros nas Amostras 3 e 4, prejudicou a avaliação das propriedades mecânicas de dureza e tenacidade à fratura. A necessidade de estudar a sinterização sem pressão, deve-se à forte presença de fase líquida oriunda, principalmente, do aço-rápido, que pode provocar vazamento de material em relação ao molde. De todo modo, os resultados contribuem para avançar com o desenvolvimento e aplicação de um FGM com propriedades que não podem ser conseguidas com materiais homogêneos.

\section{Agradecimentos}

Os autores agradecem a CAPES, FAPES (083/2019 e 144/2020) pelo fomento à pesquisa.

\section{Referências}

[1] TRENT, E.M.; WRIGHT, P.K. Metal cutting. 4. Ed. Boston: Butterworth-Heinemann. 2000. 446 p.

[2] Industry Arc. Cutting Tools Market, Forecast (2020 2025). Disponível em: <https://www.industryarc.com/Report/16304/cuttingtools-market>. Acesso em: 22 junho 2020.
[3] UPADHYAYA, G. S. Materials Science of cemented carbides - an overview. Mat. and Design, v. 22, p. 483489, 2001.

[4] ERIKSSON, M.; RADWAN, M.; SHEN, Z. 2013. Spark plasma sintering of WC, cemented carbide and functional graded materials. Int. Jour. of Refractory Metals and Hard Materials, 36, p. 31-37.

[5] BAYER, A.M.; BECHERER, B.A. 1989. High-speed tool steels. ASM Handbook: Machining. ASM International, v. 16, 944 p.

[6] CHAUS, A.S.; BRACÍK, M.; SAHUL, M.; DOMÁNKOVÁ, M. 2019. Microstructure and properties of M2 high-speed steel cast by the gravity and vacuum investment casting. Vacuum, 162, p. 183-198.

[7] MACHADO, A.R.; ABRÃO, A.M.; COELHO, R.T.; DA SILVA, M.B. Teoria da Usinagem dos Materiais. 3르. São Paulo: Edgard Blücher. 2015. 408 p.

[8] MOTT, M.; EVANS, J. R. G. Zirconia/alumina functionally graded material made by ceramic ink jet printing. Materials Science and Engineering A, v. 271, p. 344-352, 1999.

[9] YANG, K.; FENG, W. Z.; PENG, H. F.; LV, J. A new analytical approach of functionally graded material structures for thermal stress BEM analysis. International Communications in Heat and Mass Transfer, v. 62, p. 2632, 2015.

[10] KAWASAKI, A.; WATANABE, R. Concept and P/M fabrication of functionally gradient materials. Ceramics International, v. 23, p. 79-83, 1997.

[11] MA, J.; TAN, G.E.B. 2001. Processing and characterization of metal-ceramics functionally gradient materials. Journal of Materials Processing Technology, 113 , p. $446-449$.

[12] UDUPA, G.; RAO, S.S.; GANGADHARAN, K.V. Functionally Graded Composite Materials: An Overview. Procedia Materials Science, v. 5, p. 1291-1299, 2014.

[13] TOKITA, M. 2000. Mechanism of spark plasma sintering. In: Proceedings of 2000 Powder Metallurgy World Congress, Kyoto, Japan, p. 729-732.

[14] GILLIA, O.; CAILLENS, B. 2011. Fabrication of a material with composition gradient for metal/ceramic assembly. Powder Technology, 208, p. 355-366.

[15] BRASCHI, G.; EIRAS, J.A.; BARBOSA, P.A.; DE ROSSI, W.; CARNEIRO, M.B. 2017. Desenvolvimento de material em gradação funcional de metal duro e aço rápido para ferramenta de corte. Anais do IX Congresso Brasileiro de Engenharia de Fabricação. In: Congresso Brasileiro de Engenharia de Fabricação. ABCM, 2017.

[16] CARNEIRO, M.B; MACHADO, I.F.; RODRIGUES, D. 2011. Analysis of Sintering Parameters by SPS on Cemented Carbide. Advanced Materials Research, 223, p. 579-587.

[17] Zhao, S.; Song, X.; Zhang, J.; Liu, X. 2008. Effects of scale combination and contact condition of raw 
powders on SPS sintered near-nanocrystalline WC-Co alloy. Materials Science and Engineering A, 473, p. 323329.

[18] INTERNATIONAL ORGANIZATION FOR STANDARDIZATION. ISO 10545-3:1997 Ceramic tiles Part 3: Determination of water absorption, apparent porosity, apparent relative density and bulk density.

[19] GERMAN, R.M; PARK, S.J. Handbook mathematical relations in particulate materials processing: ceramics, powder metals, cermets, carbides, hard materials, and minerals. New York: John Wiley \& Sons, 2008. $419 \mathrm{p}$.

[20] AMERICAN SOCIETY FOR TESTING AND MATERIALS. ASTM International. ASTM C1327-
15:2008 Standard test method for Vickers indentation hardness of advanced ceramics.

[21] MEYERS, M.A.; CHAWLA, K.K. Mechanical behavior of materials. Cambridge university press, 2009.

[22] GERMAN, R.M. Sintering theory and practice. New York: John Wiley \& Sons Inc., 1996. 550 p.

[23] KIM, Y.; JO, H.; ALLEN, J.L.; CHOE, H.; WOLFENSTINE, J.; SAKAMOTO, J. 2016. The effect of relative density on the mechanical properties of hotpressed cubic $\mathrm{Li}_{7} \mathrm{La}_{3} \mathrm{Zr}_{2} \mathrm{O}_{12}$. Journal of the American Ceramic Society, 99, p. 1367-1374. 